\title{
Antitruncated Stellar Disks via Minor Mergers
}

\section{Citation}

Younger, Joshua D., T. J. Cox, Anil C. Seth, and Lars Hernquist. 2007. “Antitruncated Stellar Disks via Minor Mergers." The Astrophysical Journal 670 (1): 269-78. https://doi.org/10.1086/521976.

\section{Permanent link}

http://nrs.harvard.edu/urn-3:HUL.InstRepos:41381778

\section{Terms of Use}

This article was downloaded from Harvard University's DASH repository, and is made available under the terms and conditions applicable to Other Posted Material, as set forth at http:// nrs.harvard.edu/urn-3:HUL.InstRepos:dash.current.terms-of-use\#LAA

\section{Share Your Story}

The Harvard community has made this article openly available.

Please share how this access benefits you. Submit a story.

Accessibility 
Draft Version November 6, 2018

Preprint typeset using $\mathrm{AT}_{\mathrm{E} X}$ style emulateapj v. 08/22/09

\title{
ANTITRUNCATED STELLAR DISKS VIA MINOR MERGERS
}

\author{
Joshua D. Younger, T. J. Cox, Anil C. Seth, \& Lars Hernquist \\ Harvard-Smithsonian Center for Astrophysics, 60 Garden Street, Cambridge, MA 02138 \\ Draft version November 6, 2018
}

\begin{abstract}
We use hydrodynamic simulations of minor mergers of galaxies to investigate the nature of surface brightness excesses at large radii observed in some spiral galaxies: antitruncated stellar disks. We find that this process can produce the antitruncation via two competing effects: (1) merger-driven gas inflows that concentrate mass in the center of the primary galaxy and contract its inner density profile; and (2) angular momentum transferred outwards by the interaction, causing the outer disk to expand. In our experiments, this requires both a significant supply of gas in the primary disk, and that the encounter be prograde with moderate orbital angular momentum. The stellar surface mass density profiles of our remnants both qualitatively and quantitatively resemble the broken exponentials observed in local face-on spirals that display antitruncations. Moreover, the observed trend towards more frequent antitruncation relative to classical truncation in earlier Hubble types is consistent with a merger-driven scenario.

Subject headings: galaxies: kinematics and dynamics, galaxies: interactions, galaxies: formation, galaxies: evolution, galaxies: spiral, galaxies: structure, methods: $n$-body simulations
\end{abstract}

\section{INTRODUCTION}

Through the pioneering studies of Patterson (1940) and de Vaucouleurs (1959), it was first recognized that the stellar disks of most spiral galaxies are wellapproximated by an exponential surface brightness profile. However, van der Kruit (1979) later found that in many cases this description breaks down at large radius, where the disk surface density appears to be truncated (see Pohlen \& Truiillo 2006, and references therein).

Many studies of truncated disks have examined the surface brightness profiles of local edge-on systems (van der Kruit 1979; Barnaby \& Thronson 1992; Barteldrees \& Dettmar 1994; Pohlen et al. 2000; de Griis et al. 2001; van der Kruit 2001; Kregel et al. 2002; Florido et al. 2006a b) and at higher redshifts $z \sim 1$ (Pérez 2004; Trujillo \& Pohlen 2005). This choice of inclination facilitates detection of truncations, but is subject to potential biases owing to the effects of dust extinction and line-of-sight integration (see, e.g., Pohlen \& Truijillo 2006). Observations of face-on systems, which mitigate these complications, were also successful at detecting truncation, but found that the surface brightness profiles are better represented by a broken exponential than a hard break (Pohlen et al. 2002; Erwin et al. 2005; Pohlen \& Trujillo 2006). Hunter \& Elmegreen (2006) also note the existence of double-exponentials in their sample of very late-type spirals and dwarfs.

These studies also uncovered a broad range of behaviors, including some disks that follow a pure exponential profile out to very large radius (Bland-Hawthorn et al. 2005; Pohlen \& Truiillo 2006), and - as Erwin et al. (2005) first observed - some that are antitruncated, with an excess surface brightness relative to an exponential profile fitted to the inner disk (Erwin et al. 2005; Pohlen \& Truiillo 2006; Pohlen et al. 2007; Erwin et al.

Electronic address: jyounger@cfa.harvard.edu
2007). These extended stellar disks dominate the light past 4-6 scale lengths, and have flatter profiles with scale lengths that are $\sim 50 \%$ larger than the inner disk.

A number of authors have proposed theoretical explanations for truncated disks. Dynamical arguments for star-formation thresholds have been successful in motivating the locations of truncations (Kennicutt 1989; Schaye 2004; Naab \& Ostriker 2006). However, observations of ultraviolet emission (Thilker et al. 2005; Gil de Paz et al. 2005, 2006; Boissier et al.|2006), young stellar populations (Cuillandre et al. 2001), and HII regions (Ferguson et al. 1998) in extended disks suggest that there is indeed star formation occurring beyond implied thresholds. More recently, star formation models including a variety of triggering mechanisms (gravitational instabilities, spiral wave shocks, and stellar and turbulent compression; Elmegreen \& Hunter 2006) and $\mathrm{N}$-body simulations of angular momentum redistribution via bar instabilities (Debattista et al. 2006) have been more successful at explaining classically truncated disks. Despite these successes, theoretical mechanisms for producing antitruncated disks have received comparatively little attention.

While it is clear that secular processes can influence disk structure and may produce truncated stellar disks, it has also become apparent that disk galaxies exist within a hierarchical universe, in which mergers are a frequent occurrence (Lacev \& Cole 1993; Somerville \& Kolatt 1999; Somerville et al. 2000). Furthermore, these mergers are likely to play an important role in shaping the appearance of galaxies. This is certainly true of collisions between spiral galaxies of equal mass, so called major mergers, which have been suggested as the dominant formation mechanism for present-day elliptical galaxies (Toomre \& Toomre 1972; Toomre 1977; Negroponte \& White 1983; Barnes 1992; Hernquist \& Weinberg 1992; Hernquist 1993b; Silk \& Wyse 1993; Naab \& Burkert 2003; 
Robertson et al. 2006a b; Cox et al. 2006b).

There has also been considerable study of the effects of minor mergers $\left(M_{\text {prim }} / M_{\text {sec }} \gtrsim 3\right)$ on the vertical structure and dynamics of stellar disks Quinn \& Goodman 1986; Quinn et al. 1993; Walker et al. 1996; Huang \& Carlberg 1997; Sellwood et al. 1998; Velazquez \& White 1999; Font et al. 2001; Ardi et al. 2003; Brook et al. 2004, 2005, 2006; Gauthier et al. 2006; Hayashi \& Chiba 2006; Kazantzidis et al. (2007), in addition to observational evidence for past interactions with satellites as the origin of the Milky Way's thick disk (Freeman \& Bland-Hawthorn 2002; Gilmore et al. 2002; Wvse et al. 2006). Moreover, tidal structures indicative of recent minor mergers have been observed in both the Milky Way (Newberg et al. 2002; Ibata et al. 2003) and M31 (Ibata et al. 2001; McConnachie et al. 2003).

In this work, we explore the effects of minor mergers on the structure of stellar disks at large radius and find that, under certain conditions, this provides a viable physical mechanism for producing antitruncated disks. We demonstrate this process using a set of hydrodynamical simulations, which are described in $\S 2$. An overview of the merger process and specifically the dynamical response of the stellar disk during a minor merger is provided in $\S 3 . \S 4$ summarizes the surface density profile fitting procedure, and $\S 5$ and 6 describe the dependence on parameters of the interaction. In $\S[7$ we discuss our results in comparison to observations of antitruncated disks and within the context of hierarchical galaxy formation. Finally, we conclude in $\S 8$.

\section{THE SIMULATIONS}

For this study, we consider the effects of a 1:8 merger on the stellar surface density of the primary component's disk. These interactions are both cosmologically common (see, e.g., Lacey \& Cole 1993; Somerville \& Kolatt 1999; Somerville et al. 2000) and kinematically important enough to play a significant role in determining the appearance of most present-day stellar disks, while at the same time largely preserving the overall disk structure (see, e.g., Quinn et al. 1993; Walker et al. 1996; Velazquez \& White 1999; Font et al. 2001; Kazantzidis et al. 2007).

We consider the idealized case of an isolated interaction, in contrast to much work on disk galaxy formation done in a full cosmological context (Font et al.|2001; Ardi et al.|2003; Brook et al. 2004, 2005; Gauthier et al. 2006; Kazantzidis et al. 2007). The cosmological approach has the relative advantage of a more realistic accretion history. However, the isolated interactions analyzed here offer the alternative benefit of examining the individual effects of a single encounter, and allow us to efficiently sample the parameter space of interactions. Furthermore, our approach allows the simulation to be performed at much higher resolution. This helps capture the dynamical effects of minor mergers on the surface density profile at large radius, where resolution is critical.

The simulations presented in this study were performed with GADGET2 (Springel 2005), an N-Body/SPH (Smooth Particle Hydrodynamics) code using the entropy conserving formalism of Springel \& Hernquist (2002). We include the effects of radiative cooling and star formation, tuned to fit the observed Schmidt Law
TABLE 1

Model Galaxy Parameters

\begin{tabular}{ccc}
\hline \hline & Sв & IM \\
\hline$M\left(10^{10} h^{-1} M_{\odot}\right)$ & 95 & 12 \\
$V_{c}\left(\mathrm{~km} \mathrm{~s}^{-1}\right)$ & 160 & 80 \\
$c$ & 9 & 12 \\
$h_{D}\left(h^{-1} \mathrm{kpc}\right)$ & 4.1 & 1.6 \\
$M_{D} / M$ & 0.05 & 0.05 \\
$N_{H}$ & $1.0 \times 10^{6}$ & $1.3 \times 10^{5}$ \\
$N_{B}$ & $8 \times 10^{5}$ & $4.3 \times 10^{4}$ \\
\hline \hline
\end{tabular}

TABLE 2

Simulation Orbital Parameters

\begin{tabular}{cccc}
\hline \hline Name & $i$ & $f_{g}$ & $\begin{array}{c}R_{p} \\
\left(h^{-1} \mathrm{kpc}\right)\end{array}$ \\
\hline \hline Sв0Im30RP1 & $30^{\circ}$ & 0.0 & 5.0 \\
Sв2Im30RP1 & $30^{\circ}$ & 0.2 & 5.0 \\
SB4Im30RP1 & $30^{\circ}$ & 0.4 & 5.0 \\
SB8Im30RP1 & $30^{\circ}$ & 0.8 & 5.0 \\
\hline SB2Im0RP1 & $0^{\circ}$ & 0.2 & 5.0 \\
SB2Im90RP1 & $90^{\circ}$ & 0.2 & 5.0 \\
Sв2Im150RP1 & $150^{\circ}$ & 0.2 & 5.0 \\
Sв2Im180RP1 & $180^{\circ}$ & 0.2 & 5.0 \\
\hline SB2Im30RP2 & $30^{\circ}$ & 0.2 & 2.5 \\
SB2Im30RP3 & $30^{\circ}$ & 0.2 & 10.0 \\
\hline \hline
\end{tabular}

(Schmidt 1959; Kennicutt 1998). We also incorporate a sub-resolution multi-phase feedback model of the interstellar medium (ISM) (Springel \& Hernquist 2003) softened $\left(q_{E O S}=0.25\right)$ such that the mass-weighted ISM temperature is $\sim 10^{4.5}$ - and sink particles representing supermassive black holes that can accrete gas and release isotropic thermal energy to the surrounding medium (Springel et al. 2005b). For further details on the progenitor galaxy models, we refer to Springel et al. (2005a), and to other work done as part of a larger study of the effects of galaxy interactions on the formation and evolution of galaxies (Di Matteo et al. 2005; Hopkins et al. 2005a,b, d, 2006a b, c, 2007c; Robertson et al. 2006a, b; Cox et al. 2006a, b. c).

A summary of the galaxy models used in the simulations is provided in Table1, including the total (baryons and dark matter) mass $M$, circular rotation velocity $V_{c}$, concentration parameter $c$, initial disk scale length $h_{D}$, disk (stars and gas) mass fraction $M_{D} / M$, number of dark matter particles in the halo $N_{H}$, and baryonic (stars and gas) particles $N_{B}$. Both are designed to be representative of their eponymous local hubble types (see e.g., Roberts \& Haynes 1994).

The different encounter configurations considered are summarized in Table 2. We assume zero-energy parabolic orbits $(e=1)$, as motivated by cosmological simulations (Benson 2005; Khochfar \& Burkert 2006), with radius of pericenter $R_{p}$, orbital inclination $(i)$, and primary disk gas fraction $f_{g}$. For the primary disk $(\mathrm{SB})$, we consider $20 \%$ gas disks $\left(f_{g}=0.2\right)$, which are intended to be representative of disks in the local universe (McGaugh \& de Blok 1997; Bell \& de Jong $2000)$, and higher gas fractions $\left(f_{g}=0.4,0.8\right)$ which are consistent with both the more gas-rich local systems (McGaugh \& de Blok 1997) and high redshift $(z \sim 2)$ 


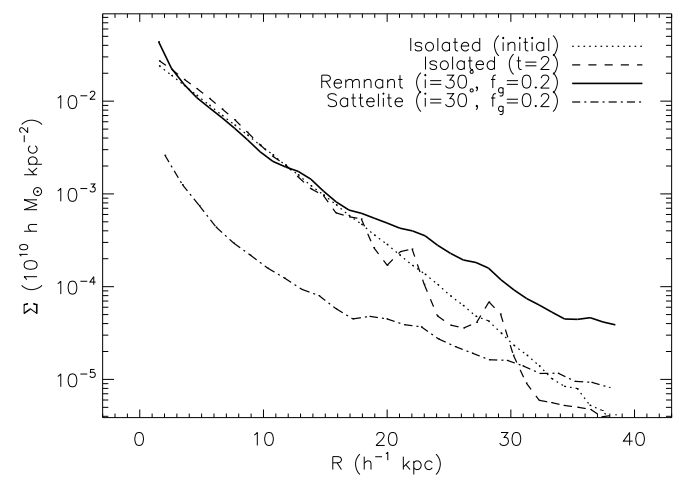

FIG. 1.- Stellar surface density profiles $(\Sigma)$ as a function of radial distance from the stellar center of mass $(R)$ for an isolated $20 \%$ gas disk SB2, and the remnant SB2Im30RP1. Included are the initial SB disk (dotted), an SB2 disk evolved in isolation for $2 h^{-1}$ Gyr (dashed), and the SB2Im30RP1 remnant (solid). Also shown is contribution of stars from the secondary disk to the total stellar surface density in the remnant (dash-dot).

spirals (Erb et al. 2006). The secondary disk has a fixed gas fraction of $40 \%\left(f_{g}=0.4\right)$ - with the exception of the purely collisionless interaction SB0Im30RP1 which has $f_{g}=0$ - and is consistent with observations of dwarf galaxies and low-mass disks in the local universe (Schombert et al. 2001; Geha et al. 2006). The initial spins of the two disks are not aligned, and are the same in all our simulations.

\section{DYNAMICAL RESPONSE OF THE STELLAR DISK TO A MINOR MERGER}

We find that minor mergers can create antitruncated stellar disks in face-on spirals. To examine this effect in detail and illustrate some of the generic features of a minor merger, we concentrate on SB2Im30RP1 and its collisionless counterpart SB0Im30RP1. In all cases, the stellar mass surface density profiles of the remnant are measured $1 h^{-1}$ Gyr after the final coalescence - or several orbital periods at the half mass radius - to allow the remnant disk to reach a state of approximate dynamical equilibrium.

In our experiments, antitruncations are produced only when there is a significant supply of gas in the primary disk. The driving physical mechanism for producing this outcome represents a competition between merger driven inflows and transfer of angular momentum to large radius in the remnant stellar disk; i.e., gas moves inwards while stars move outwards. This effect is manifest in the changes induced in both the gravitational potential and angular momentum profile during the encounter.

In Figure 1, we present the stellar mass surface density profiles for SB2Im30RP1, compared to both the initial primary disk SB2 and the same initial disk evolved in isolation for $2 h^{-1} \mathrm{Gyr}$, as a function of radial distance from the stellar center of mass $R$. The primary disk is stable; when it is evolved in isolation over several orbital periods, the inner scale length is only marginally shorter owing to preferential star formation occurring near the center (SFR $\sim \rho_{g}^{1.4}:$ Kennicutt 1998). At large radius, there is some fluctuation of the evolved, isolated disk about the initial stellar mass surface density. This owes to Poisson noise arising from low particle counts at large $R$ and the development of spiral structure from numerical

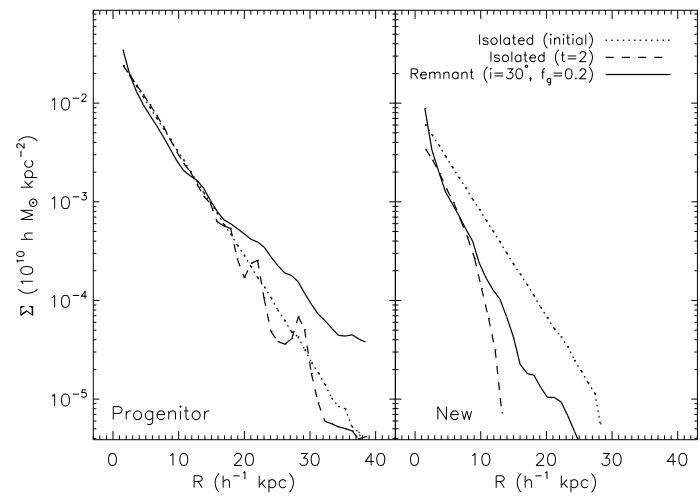

Fig. 2.- Same as Figure 1 but split into the two component stellar populations: "progenitor" stars which are present at the start of the simulation, and "new" stars which are formed during the interaction. The dotted line in the right panel shows the initial gas distribution.

noise associated with the discretized dark matter halo (Hernquist 1993a).

The surface density profile of the SB2IM30RP1 merger remnant shown in Figure 1 displays three key features. First, within $4 h^{-1} \mathrm{kpc}$, the surface density profile is steep, indicative of a bulge component produced by the merger. Second, the surface density profile from $4-20$ $h^{-1} \mathrm{kpc}$ is nearly identical to the primary disk. Third, beyond $20 h^{-1} \mathrm{kpc}$, there is a clear excess of surface density relative to the initial disk of the primary.

The excess surface density in the outer profile of the remnant Sв2Im30RP1, i.e., the antitruncation of its disk, qualitatively - and, as we will see in $\S 5$ and $\S 6$, quantitatively - resembles the broken exponentials of Pohlen \& Truiillo (2006), with an inner scale length close to that of the initial disk. In Figure 2, we separate out the "progenitor" stellar particles that are initialized with the disks and "new" stellar particles formed from the gas during the interaction. We find that the antitruncation is dominated by progenitor stars. An antitruncated disk in Sв2Im30RP1 is produced independent of a fitted profile; a robust result with respect to any fitting procedure. Separating out the stellar particles that originate in the secondary stellar disk (see Figure 1), we find that the increased outer surface density $\left(R \gtrsim 20 h^{-1} \mathrm{kpc}\right)$ is dominated by progenitor stars from the primary disk that have been transferred to larger radius by the interaction. Furthermore, we find that these large $R$ features in the profile are rotationally supported - their median circular velocity in circular annuli is $\sim 0.8-0.9$ times the Keplerian orbital velocity at that radius - and thus long-lived. We confirm this by evolving our remnant in isolation for $\sim 10^{10}$ years, and find that the antitruncation is not a transient feature.

The surface density profiles shown in Figure 3 demonstrate that the collisionless interaction Sв0Iм30RP1 displays no antitruncation in the stellar mass surface density profile of its remnant. Rather, its surface density profile, which has been tilted, increasing the scale length at all radii, does not have a well-defined break. Features at large $R$ are, as in the previous case, also rotationally supported.

Although there is some evidence for bulge formation in Sв2Im30RP1 at $R \lesssim 2 h^{-1} \mathrm{kpc}$, Sв0Im30RP1 shows 


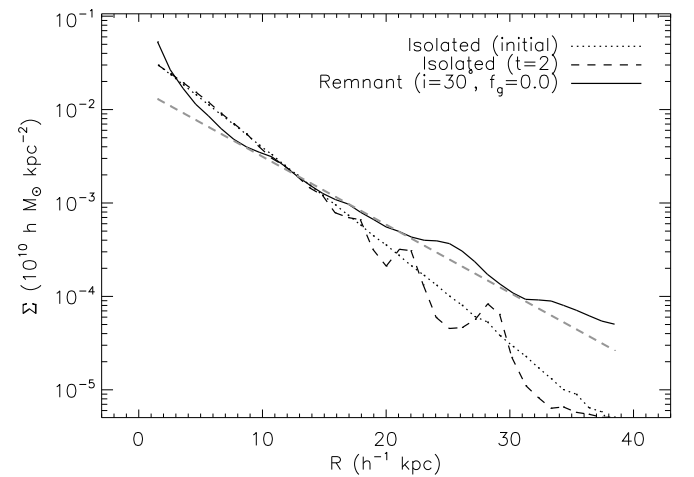

FIG. 3.- Stellar surface density profiles $(\Sigma)$ as a function of radial distance from the stellar center of mass $(R)$ for an isolated collisionless disk SB0, and the collisionless remnant SB0Im30RP1. Included are the initial Sв disk (dotted), an Sв0 disk evolved in isolation for $2 h^{-1}$ Gyr (dashed), and the SB0Im30RP1 remnant (solid). The grey dashed line is an exponential fit to the collisionless remnant Sв0Iм30RP1 for $R>10 h^{-1} \mathrm{kpc}$, meant to highlight that this profile, in contrast to the $20 \%$ gas remnant SB2IM30RP1 (see Figure 1), would not likely be observed as significantly antitruncated

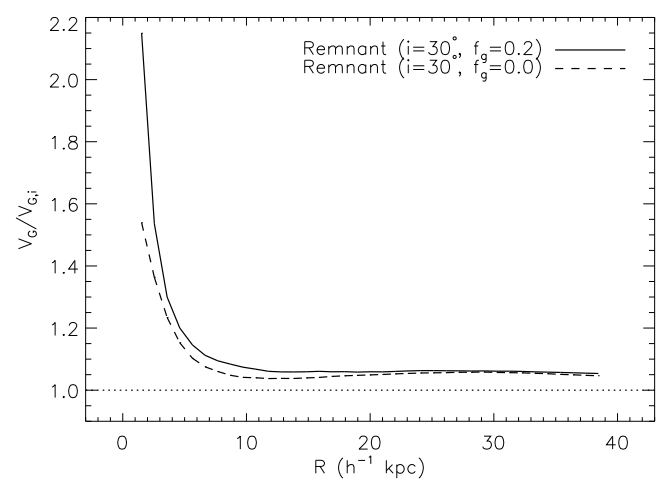

FIG. 4.- Gravitational potential relative to the initial disk, as a function of radial distance from the stellar center of mass $(R)$, for the Sв2Im30RP1 remnant (solid) and the collisionless Sв0Im30RP1 remnant (dashed). We find that dissipation efficiently deepens the central potential of the remnant relative to the initial disk.

a much more pronounced bulge which remains prominent out to larger radii. This is expected; phase space conservation in a collisionless interaction leads to lower phase space densities in the core of the collisionless remnant, and accordingly a more diffuse spheroid component (Hernquist et al. 1993). While bulge growth via minor mergers is a topic worthy of further study, we postpone a detailed analysis to future work and point out the qualitative difference between the surface density profiles of Sв2Im30Rp1 and SB0Im30Rp1.

To investigate the physical processes driving the antitruncation in our simulations, we first consider the gravitational potential as a function of radial distance from the stellar center of mass of SB0IM30RP1, and SB2Im30Rp1 as compared to the initial disk in Figure 4. For certain orbits, a minor merger can drive nuclear inflows of gas (Hernquist 1989; Mihos \& Hernquist 1994; Hernquist \& Mihos 1995), fueling a centrally concentrated starburst and creating a deeper potential well there owing to the effects of gas disspation (see Figure21). This deeper potential will contract the remnant profile,

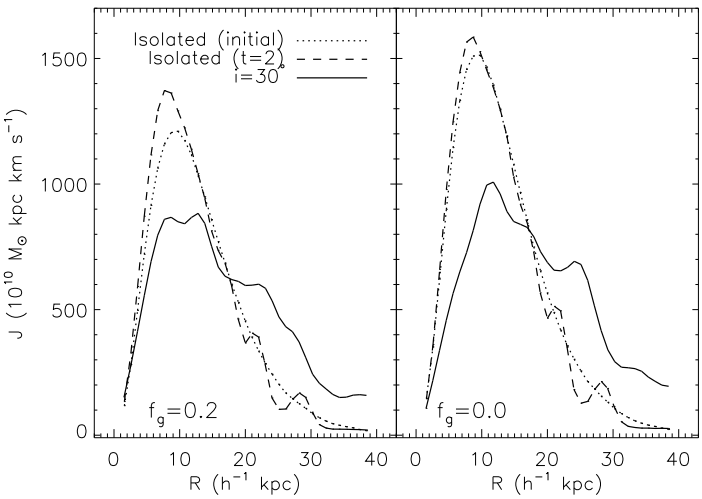

Fig. 5.- Total angular momentum in the stellar disk, as a function of radial distance from the stellar center of mass $(R)$. Shown are: the initial disk (dotted), an isolated disk evolved for $2 h^{-1}$ Gyr (dashed), and (solid) the remnant for both a gas fraction of $f_{g}=0.2$ (left: Sв2 disk and Sв2Iм30RP1 remnant) and a collisionless interaction (right: SB0 disk and SB0Im30Rp1 remnant). We find that angular momentum is transferred to the outer disk, which increases its scale length relative to the inner disk, and creates the antitruncation.

counteracting the broadening of the profile owing to angular momentum transfer and maintaining an inner scale length similar to the initial primary disk. Furthermore, the newly formed stars will be more concentrated than the progenitor stars (see Figure 2), which will also tend to contract the inner scale length and populate the stellar mass surface density at small $R$.

At the same time, the interaction transfers angular momentum and stellar mass to the outer disk. In Figure 5. we show the total angular momentum in circular annuli as a function of $R$. In both SB2Im30RP1 and SB0Im30RP1, the angular momentum at large $R$ is nearly double that of the initial and evolved disks; this transfer occurs whether or not gas is included. In SB2Im30RP1, the magnitude of the angular momentum in shells at $R<10 h^{-1} \mathrm{kpc}$ is also higher, owing to the more efficient inflows generated by dissipation during the interaction. In the collisionless case, because the inner potential is not as deep, the disk expands more uniformly in response to this transfer, and therefore does not show an antitruncation. Thus, the antitruncation at large $R$ results from expansion of the outer disk - similar to that first noted by Quinn et al. (1993) - in response to a net transfer of angular momentum. When gas is present, the inner potential is deep enough to contract this inner profile and maintain an inner scale length similar to that of the initial primary disk.

\section{FITTING THE SURFACE DENSITY PROFILE}

In $\S$ 3, we find antitruncated stellar disks independent of the fitted surface density profile. However, to facilitate comparison to the observational constraints on antitruncated disks, we fit profiles to the stellar mass density profiles of our remnants, projected face- ${ }^{-}$, and include both progenitor and new stars. The fit is performed, as in $\S\left[1 h^{-1}\right.$ Gyr after the final coalescence, so the remnant reaches a state of approximate dynamical equilibrium.

Following Pohlen \& Truillo (2006), we mask out the inner $5 h^{-1} \mathrm{kpc}$ of the disk and first fit an exponential profile (Patterson 1940; Freeman 1970) with scale length $h_{D 1}$ to the inner disk, then fit a second exponen- 
TABLE 3

Fitted Surface Density Profiles

\begin{tabular}{cccc}
\hline \hline Name & $\begin{array}{c}h_{D 1} \\
\left(h^{-1} \mathrm{kpc}\right)\end{array}$ & $\begin{array}{c}h_{D 2} / h_{D 1} \\
\left(h^{-1} \mathrm{kpc}\right)\end{array}$ & $R_{b r} / h_{D 1}$ \\
\hline \hline Sв0Im30RP1 & 5.99 & $\ldots$ & $\ldots$ \\
Sв2Im30RP1 & 4.00 & 1.69 & 3.27 \\
Sв4Im30RP1 & 3.54 & 1.85 & 3.33 \\
SB8Im30RP1 & 3.95 & 1.26 & 3.85 \\
\hline Sв2Im0RP1 & 3.38 & 1.72 & 2.47 \\
Sв2Im90RP1 & 4.10 & 1.24 & 2.64 \\
Sв2Im150RP1 & 4.64 & 1.61 & 5.45 \\
Sв2Im180RP1 & 4.22 & 1.27 & 3.49 \\
\hline Sв2Im30RP2 & 3.70 & 1.80 & 3.10 \\
Sв2Im30RP3 & 4.28 & 1.61 & 2.87 \\
\hline \hline
\end{tabular}

tial profile with scale length $h_{D 2}$ to the outer disk ${ }^{1}$. The break radius $R_{b r}$ is defined at the intersection of the inner and outer disk profiles. The fits are performed using a Levenberg-Marquardt least-squares minimization routine, with bins weighted by the Poisson error $\left(\sigma_{i} \sim \Sigma_{i}\right)$. The results are tabulated in Table 3 . We note as a caveat that the values of the parameters in our fits are somewhat sensitive to the manner in which the bins are weighted. However, using slightly different weights, such as "flux" $\left(\Sigma r^{2}\right)$, does not qualitatively affect our results.

Since we are considering the stellar mass distribution out to large radius, resolution effects are particularly important. We note that all of our simulations have more than $1.5 \times 10^{4}$ stellar particles in the "outer" disk $\left(4 h_{D 1}<R<10 h_{D 1}\right)$, and a majority have $\gtrsim 3.0 \times 10^{4}$ over the same range. Furthermore, tripling the number of particles did not change the surface density profile at large radius ( $\left.R \gtrsim 20 h^{-1} \mathrm{kpc}\right)$ by more than $15 \%$. Therefore, we find that our resolution is sufficient to make robust claims about the the stellar surface mass density profiles at large radius.

\section{DEPENDENCE ON THE GAS CONTENT}

Because antitruncation appears to be a dissipational effect, we expect the degree and location of the break to depend on the gas content of the primary disk. Therefore, we perform a set of experiments varying the gas fraction of the primary disk, while holding the orbital parameters fixed. In Figure 6] we present the stellar surface mass density profiles of the remnant, including both the progenitor and new stellar particles, for the four different gas fractions listed in $\S 2$. In addition, we show the broken exponential disk profiles listed in Table 3

We find that Sв0Im30Rp1 - the collisionless interaction, see also Figure 3- is not well-fitted by a broken exponential as in Erwin et al. (2005) and Pohlen \& Truiillo (2006), and therefore would not be observed to be antitruncated. Rather, the scale length increases to $h_{D 1} \approx$ $6 h^{-1} \mathrm{kpc}$, relative to $h_{D}=4.14 h^{-1} \mathrm{kpc}$ initially, with a substantial bulge-like component following an $R^{1 / 4}$ (de Vaucouleurs 1959) profile, which is shown in the fit presented in Figure 6 .

Figure 7 shows that the inner potential is deeper - and therefore the inner scale length shorter - for increased gas

\footnotetext{
1 The fitting range for the outer disk was set by an initial guess for $R_{b r}$. We found that the fitted value for $R_{b r}$ and the outer disk parameters were largely insensitive to this choice.
}

fractions (see Table 3). The antitruncation is strongest at $f_{g}=0.2$ and 0.4 , while for the highest gas fraction $f_{g}=0.8$, the relative scale length $h_{D 1} / h_{D 2}$ is significantly flatter, resulting in a less pronounced break. This is related to the composition of the initial disk; very gas rich disks have fewer stars remaining in the outer disk after the initial inflow of gas. Therefore, as the outer disk expands, less stellar mass resides at large radii.

\section{DEPENDENCE ON THE ORBITAL PARAMETERS}

Since the processes that create the antitruncation are dynamical, the degree and location of the break should be sensitive to the orbital parameters of the encounter. To investigate this, we vary the orbital parameters - the orbital inclination $i$ and radius of pericenter $R_{p}$-holding the gas content of the primary disk fixed.

\subsection{Orbital Angular Momentum}

To test the ability of the deeper potential to mitigate against the expansion of the inner disk owing to angular momentum transfer, we vary the total angular momentum of the secondary's orbit, while fixing the orbital inclination. This is done by adjusting the location of the pericenter of the secondary's orbit.

We find that the antitruncation is largely insensitive to increasing the orbital angular momentum. Figure 8 shows the surface density profiles for three different radii of pericenter $\left(R_{p}\right)$ spanning a factor of four in orbital angular momentum. The broken exponential fits are listed in Table 3. Though the inner scale length does increase over this range - despite a deeper inner potential (see Figure 9) - it does so by less than $20 \%$. Over the same range, the break radius and relative scale lengths decrease by less than $10 \%$. Therefore, we expect that at fixed inclination, most orbits would create similar antitruncations.

\subsection{Orbital Inclination}

Varying the orbital inclination of the interaction introduces two competing effects. First, prograde minor mergers are more efficient than retrograde mergers at coupling to the rotation of the primary disk. At the same time, coplanar minor mergers are more efficient at transferring angular momentum to the stellar orbits, inducing bar formation, and centrally concentrating gas and stars. As a result, the closer the interaction is to coplanar, the deeper the remnant's inner potential. We present results for five different inclinations, as outlined in $\S 2$ and Table 2, in Figures 10 and 11.

We first consider the prograde and polar interactions Sв0Im0Rp1, Sв0Im30Rp1, and Sв0Im90Rp1 - to illustrate the combined effects of the merger-driven inflow. As the inclination increases from $i=0$ (coplanar prograde) to $i=90$ (polar), the potential at small scale radius is shallower (see Figure 11). Accordingly, the inner scale radii of Sв0Im0RP1, Sв0Im30RP1, and Sв0Im90RP1 are successively larger with flatter relative outer to inner scale lengths $h_{D 2} / h_{D 1}$. As a result, the antitruncation is less pronounced for increasing $i$.

When the interaction is retrograde, as with Sb150Im0Rp1 and SB180Im0Rp1, the orbital frequency of the secondary is out of resonance with the orbits of particles in the inner disk. This makes the 


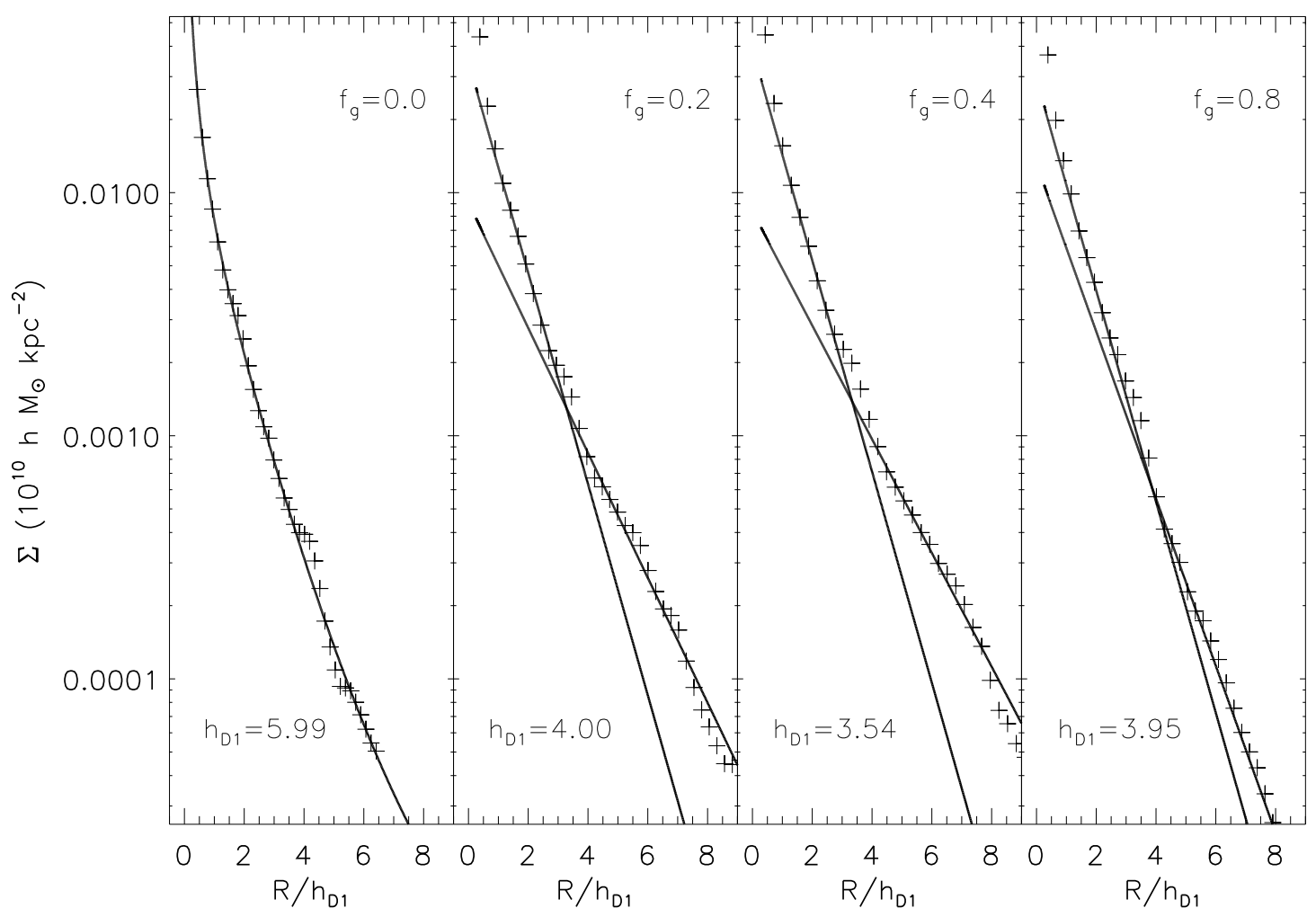

FIG. 6.- Stellar mass surface density of the remnant as a function of radial distance from the center of mass $R$ in units of the inner scale length $h_{D 1}$ (see Table 3 for fitted values), and its dependence on the initial gas fraction of the primary disk $f_{g}$ : from left to right $f_{g}=0.0$ (Sв0Im30Rp1), 0.2 (Sв2Im30Rp1), 0.4 (Sв4Im30Rp1), and 0.8 (Sв8Im30Rp1). We include both the binned simulation data (crosses), and fitted disk profiles (solid line). The collisionless $\left(f_{q}=0.0\right)$ remnant is not well-described by a double exponential, and instead has been fit with a combination disk and de Vaucouleurs (1959) profile.

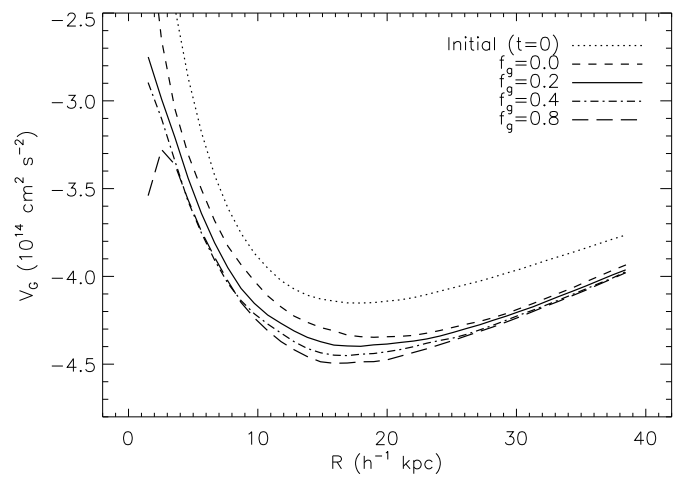

FIG. 7.- Response of the gravitational potential to a minor merger as a function of radial distance from the stellar center of mass $R$, and its dependence on the initial gas fraction of the primary disk $f_{g}: f_{g}=0.0$ (dashed, SB0IM30RP1), 0.2 (solid, Sв2Im30Rp1), 0.4 (dash-dot, Sв4Im30Rp1), and 0.8 (long dash, Sв8Im30Rp1). The dotted line shows the initial potential of the primary disk.

encounter much less efficient at transferring angular momentum to the outer disk. As a result, though there are antitruncations in our retrograde experiments, they are far less pronounced than those in prograde encounters. Therefore, our simulations suggest that prograde minor mergers will be most effective at producing antitruncations.
We find that minor mergers can create antitruncated stellar disks in face-on spiral galaxies, and that this antitruncation is produced by a competition between merger driven inflows of gas and transfer of angular momentum to large $R$ in the remnant stellar disk that moves stars outwards. Because this process requires gaseous inflows, antitruncated stellar disks are produced only when gas dissipation and star formation are included. Moreover, the magnitude and location of antitruncation is related to both the gas content of the primary disk and the orbital parameters of the interaction. These features in the surface stellar mass density profile are rotationally supported, and therefore long-lived and likely to be observed in local spirals.

This merger-driven scenario for the production of antitruncated disks is supported by observations of faceon spirals that find antitruncated disks occur more frequently in earlier-type spirals and in higher density environments (Pohlen \& Trujillo 2006). The authors note that the frequency of antitruncated disks increases from $20 \%$ in $\mathrm{Sd}$ types to $50 \%$ in Sb types, while the fraction of classically truncated disks decreases from $40 \%$ to $10 \%$ over the same range. This agrees qualitatively with minor mergers as the physical mechanism driving disk antitruncation: spirals in higher density environments are more likely to have undergone minor mergers which create systematically earlier Hubble types (Naab \& Burkert 2003). At the same time, both Erwin et al. (2005) and Pohlen \& Trujillo (2006) show observational evidence for

\section{DISCUSSION}




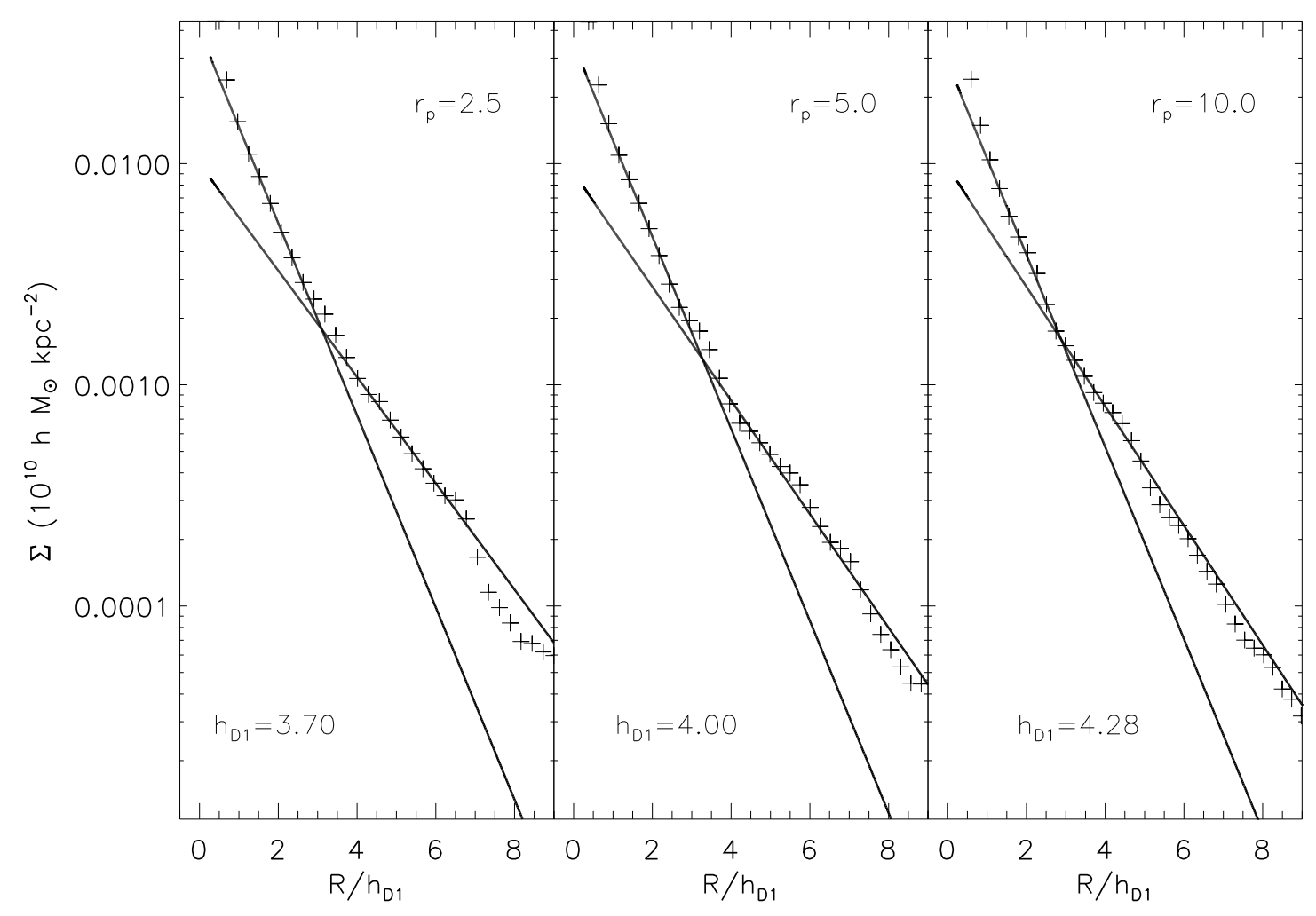

FIG. 8. - Stellar surface mass density of the remnant as a function of radial distance from the center of mass $R$ in units of the inner scale length $h_{D 1}$ (see Table 3 for fitted values), and its dependence on the radius at pericenter $R_{p}$ of the orbit of the secondary: from left to right $R_{p}=2.5$ (Sв2Im30Rp2), 5.0 (Sв2Im30RP1), and 10.0 (Sв2Im30Rp3) $h^{-1} \mathrm{kpc}$. We include both the binned simulation data (crosses), and fitted disk profiles (solid line).

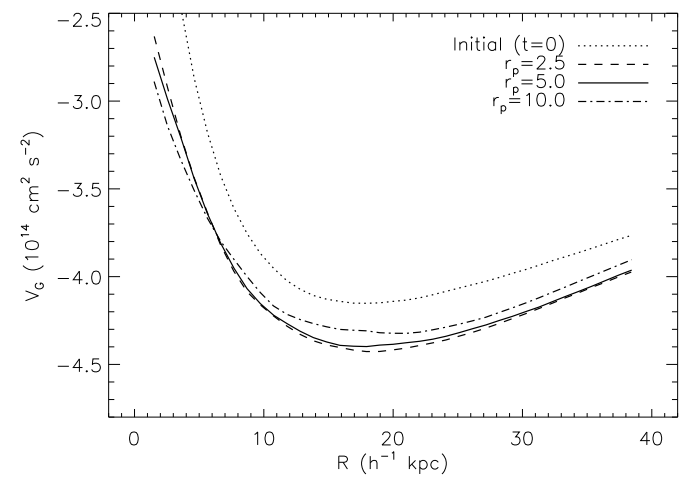

FIG. 9.- Response of the gravitational potential to a minor merger as a function of radial distance from the center of mass $R$, and its dependence on the radius at pericenter $R_{p}$ of the orbit of the secondary: $R_{p}=2.5$ (dashed, SB2Im30RP2), 5.0 (solid, Sв2Im30Rp1), and 10.0 (dot-dash, Sв2Im30Rp3) $h^{-1} \mathrm{kpc}$.

asymmetries or recent interactions in antitruncated systems, which further supports a merger-driven scenario.

Using the extended Press-Schechter formalism (Jenkins et al. 2001) and the method of Lacey \& Cole (1993) to estimate halo merger histories, and assuming the cosmology of Spergel et al. (2003), we find that SB-type halos $\left(M_{t o t} \sim 10^{12} h^{-1} M_{\odot}\right)$ are likely to experience of order one 1:8 minor merger from $z=1$ to the present-day (Hopkins et al. 2007a, b). In our simulations, strong antitruncations are produced when the orbit of the secondary is inclined $\left(0^{\circ} \lesssim i \lesssim 90^{\circ}\right)$, pro- grade, and has moderate angular momentum $\left(R_{p} \gtrsim h_{D}\right)$. So, if all orbits are distributed isotropically - i.e., equally likely in bins of $d \cos i$ - and follow the distribution of $R_{p}$ as inferred from cosmological N-body simulations (e.g., Benson 2005), then we would expect $\sim 40 \%-50 \%$ of $\mathrm{Sb}$ type spirals to have pronounced antitruncations.

At the same time, our fits agree quantitatively with the relative scale lengths $h_{D 2} / h_{D 1}$ observed by Pohlen \& Trujillo (2006) and Erwin et al. (2005). We find, however, that the break radius in our simulations is at the low end of the observed range; Pohlen \& Trujillo (2006) and Erwin et al. (2005) find $R_{b r} / h_{D 1}=3-6$ while in our simulations $R_{b r} / h_{D 1}=3-4$. This may owe either to: (1) the smaller mass of a typical galaxy in the Pohlen \& Trujillo (2006) sample, or (2) the limited range of parameter space spanned by our simulations. The observations of Pohlen \& Trujillo (2006) appear to be dominated by somewhat lower-mass spirals than the Milky-Way mass primary disk in the interactions examined here. This could potentially lead to shorter inner scale lengths (e.g., Courteau 1996; de Jong 1996), which may tend to increase the average observed $R_{b r} / h_{D 1}$ ratio. Also, our simulations sample only a small subset of the parameter space for individual interactions. The real merging history of galaxies likely involves a variety of mass fractions and multiple mergers that may produce subtly different effects. However, we find that our simulations of minor mergers generically lead to antitruncated disks for a range of orbital geometries, and therefore represent a viable mechanism for producing these features. 


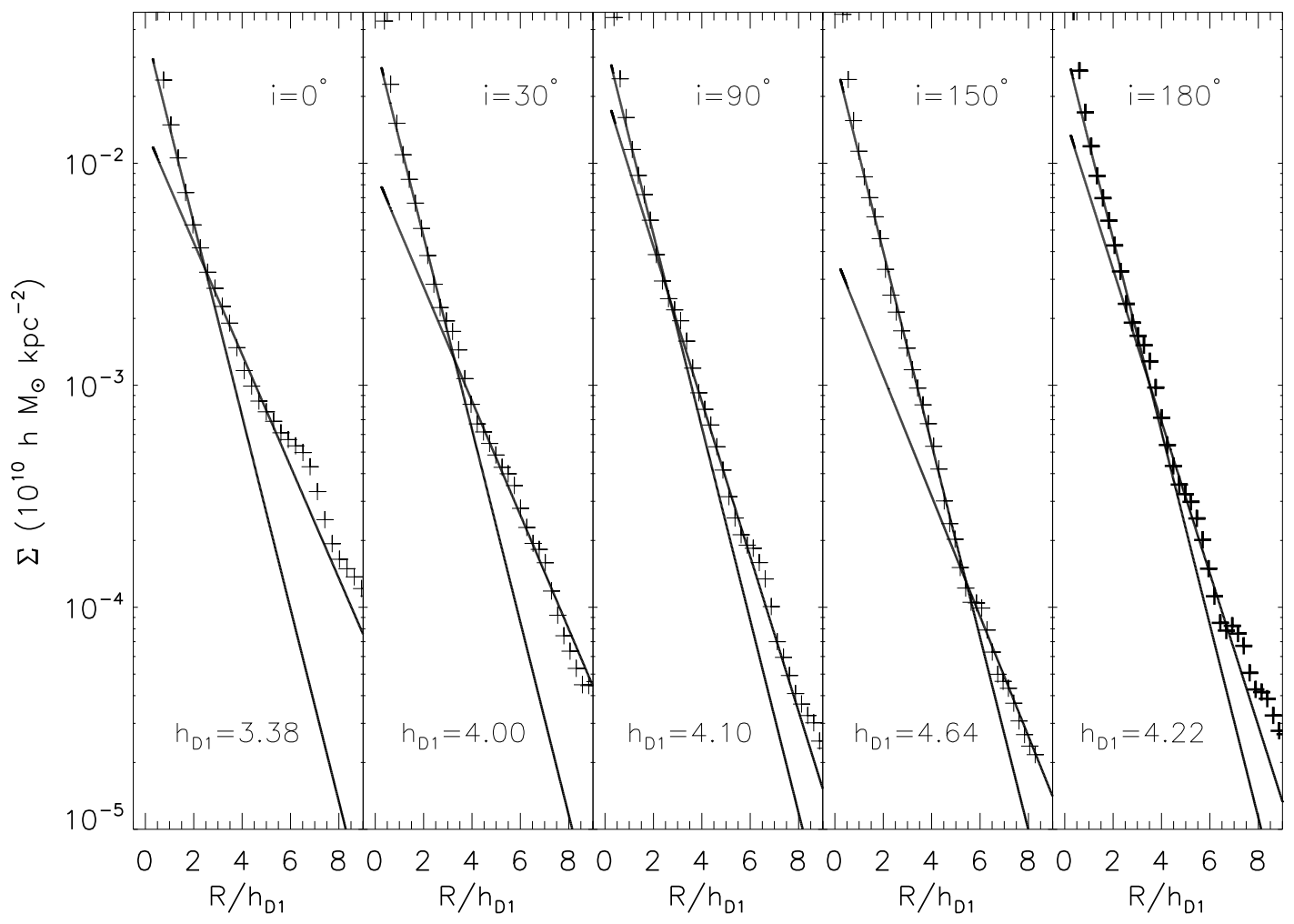

Fig. 10. - Stellar surface mass density of the remnant as a function of radial distance from the center of mass $R$ in units of the inner scale length $h_{D 1}$ (see Table 3 for fitted values), and its dependence on the orbital inclination $i$ of the interaction: from left to right $i=0$ (coplanar prograde, Sв2Im0RP1), 30 (prograde, Sв2Im30RP1), 90 (polar, Sв2Im90RP1), 150 (retrograde, Sb2Im150Rp1), and 180 (coplanar retrograde, SB2Im180RP1). We include both the binned simulation data (crosses), and fitted disk profiles (solid line).

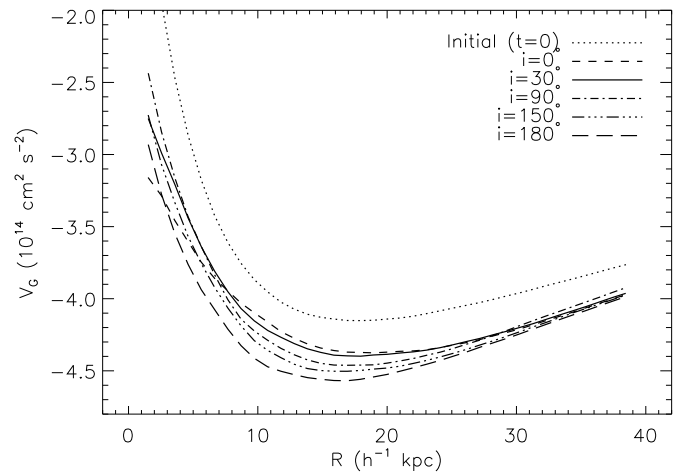

FIG. 11.- Response of the gravitational potential to a minor merger as a function of radial distance from the center of mass $R$, and its dependence on the orbital inclination $i$ of the interaction: $i=0$ (coplanar prograde, dashed, Sв2Im0RP1), 30 (prograde, solid, Sв2Im30RP1), 90 (polar, dot-dash, Sв2Im90RP1), 150 (retrograde, double dot-dash, Sв2Im150RP1), and 180 (coplanar retrograde, long dash, Sв2Im180Rp1). The dotted line represents the initial potential of the primary disk.

More locally, Ibata et al. (2005) and Ibata et al. (2007) recently observed an extended stellar disk in M31. Though it is not entirely clear whether or not this feature is preceded by a well defined break in the surface brightness profile, it is possible that the extended disk represents an antitruncation of the type observed by Erwin et al. (2005) and Pohlen \& Truijllo (2006). Ibata et al. (2005) estimate that it contains roughly $10 \%$ of the stellar mass and $30 \%$ of the angular momentum of the total stellar disk, as compared to $5 \%$ of the mass and $45 \%$ of the angular momentum in the antitruncated disk of Sв2Im30RP1. Furthermore, the kinematics - specifically, the dispersion of circular velocity lags relative to Keplerian rotation - of resolved stars in the extended disk show evidence of dynamical heating which could have been caused by a minor merger (e.g. Quinn et al. 1993; Walker et al. 1996; Velazquez \& White 1999). Therefore, although we cannot say with certainty that the extended disk of M31 represents a local example of an antitruncation, it is broadly consistent with our modeling.

\section{CONCLUSION}

We use hydrodynamic simulations to investigate minor mergers as a physical mechanism for creating antitruncated disks in face-on spirals. We find that the antitruncation is produced by two competing effects: mergerdriven gas inflows deepen the central potential and contract the inner profile, while at the same time angular momentum is transferred to large radius and causes the outer disk to expand. Because the inflows are far more efficient when gas dissipation is included, the antitruncation is produced in our experiments only when a significant gas supply is present in the initial primary disk. This effect is also only seen when the interaction is prograde, rather than polar or retrograde, with moderate $\left(R_{p} \sim h_{D}\right)$ orbital angular momentum.

Our merger-driven scenario for producing antitruncated disks yields results that agree with obser- 
vations of local face-on spirals (Erwin et al. 2005; Pohlen \& Truiillo 2006), both in terms of the parameters of the antitruncation and its frequency with Hubble type. Therefore, we find that minor mergers are a viable physical mechanism for producing antitruncated disks.

Particular thanks to the referee, Michael Pohlen, for his comments and suggestions that improved this manuscript. Also thanks to Peter Erwin, Yuexing Li, Phillip F. Hopkins, Beth Willman, and Dušan Kereš for helpful discussions. These simulations were performed at the Harvard Institute for Theory and Computation at the Smithsonian Center for Astrophysics.
Ardi, E., Tsuchiya, T., \& Burkert, A. 2003, ApJ, 596, 204

Barnaby, D. \& Thronson, Jr., H. A. 1992, AJ, 103, 41

Barnes, J. E. 1992, ApJ, 393, 484

Barteldrees, A. \& Dettmar, R.-J. 1994, A\&AS, 103, 475

Bell, E. F. \& de Jong, R. S. 2000, MNRAS, 312, 497

Benson, A. J. 2005, MNRAS, 358, 551

Bland-Hawthorn, J., Vlajić, M., Freeman, K. C., \& Draine, B. T. 2005, ApJ, 629, 239

Boissier, S. et al. 2006, astro-ph/0609071

Brook, C., Richard, S., Kawata, D., Martel, H., \& Gibson, B. K. 2006, astro-ph/0611748

Brook, C. B., Gibson, B. K., Martel, H., \& Kawata, D. 2005, ApJ, 630, 298

Brook, C. B., Kawata, D., Gibson, B. K., \& Freeman, K. C. 2004 ApJ, 612, 894

Courteau, S. 1996, ApJS, 103, 363

Cox, T. J., Di Matteo, T., Hernquist, L., Hopkins, P. F., Robertson, B., \& Springel, V. 2006a, ApJ, 643, 692

Cox, T. J., Dutta, S. N., Di Matteo, T., Hernquist, L., Hopkins, P. F., Robertson, B., \& Springel, V. 2006b, ApJ, 650, 791

Cox, T. J., Jonsson, P., Primack, J. R., \& Somerville, R. S. 2006c, MNRAS, 373, 1013

Cuillandre, J.-C., Lequeux, J., Allen, R. J., Mellier, Y., \& Bertin, E. 2001, ApJ, 554, 190

de Grijs, R., Kregel, M., \& Wesson, K. H. 2001, MNRAS, 324, 1074

de Jong, R. S. 1996, A\&A, 313, 45

de Vaucouleurs, G. 1959, Handbuch der Physik, 53, 311

Debattista, V. P., Mayer, L., Carollo, C. M., Moore, B., Wadsley, J., \& Quinn, T. 2006, ApJ, 645, 209

Di Matteo, T., Springel, V., \& Hernquist, L. 2005, Nature, 433, 604

Elmegreen, B. G. \& Hunter, D. A. 2006, ApJ, 636, 712

Erb, D. K., Steidel, C. C., Shapley, A. E., Pettini, M., Reddy, N. A., \& Adelberger, K. L. 2006, ApJ, 646, 107

Erwin, P., Beckman, J. E., \& Pohlen, M. 2005, ApJ, 626, L81

Erwin, P., Pohlen, M., \& Beckman, J. E. 2007, AJ, submitted

Ferguson, A. M. N., Wyse, R. F. G., Gallagher, J. S., \& Hunter, D. A. 1998, ApJ, 506, L19

Florido, E., Battaner, E., Guijarro, A., Garzón, F., \& Castillo-Morales, A. 2006a, A\&A, 455, 475

-. 2006b, A\&A, 455, 467

Font, A. S., Navarro, J. F., Stadel, J., \& Quinn, T. 2001, ApJ, 563, L1

Freeman, K. \& Bland-Hawthorn, J. 2002, ARA\&A, 40, 487

Freeman, K. C. 1970, ApJ, 160, 811

Gauthier, J.-R., Dubinski, J., \& Widrow, L. M. 2006, ApJ, 653, 1180

Geha, M., Blanton, M. R., Masjedi, M., \& West, A. A. 2006, ApJ, 653,240

Gil de Paz, A., Madore, B. F., Boissier, S., Swaters, R., Popescu, C. C., Tuffs, R. J., Sheth, K., Kennicutt, Jr., R. C., Bianchi, L., Thilker, D., \& Martin, D. C. 2005, ApJ, 627, L29

Gil de Paz, A. et al. 2006, astro-ph/0606440

Gilmore, G., Wyse, R. F. G., \& Norris, J. E. 2002, ApJ, 574, L39

Hayashi, H. \& Chiba, M. 2006, PASJ, 58, 835

Hernquist, L. 1989, Nature, 340, 687

-. 1993a, ApJS, 86, 389

-. 1993b, ApJ, 409, 548

Hernquist, L. \& Mihos, J. C. 1995, ApJ, 448, 41

Hernquist, L., Spergel, D. N., \& Heyl, J. S. 1993, ApJ, 416, 415

Hernquist, L. \& Weinberg, M. D. 1992, ApJ, 400, 80

Hopkins, P. F., Cox, T. J., Keres, D., \& Hernquist, L. 2007a, ApJ, submitted (astro-ph/0706.1246)

\section{REFERENCES}

Hopkins, P. F., Hernquist, L., Cox, T. J., Di Matteo, T., Martini, P., Robertson, B., \& Springel, V. 2005a, ApJ, 630, 705

Hopkins, P. F., Hernquist, L., Cox, T. J., Di Matteo, T., Robertson, B., \& Springel, V. 2005b, ApJ, 630, 716

-. 2005c, ApJ, 632, 81

-. 2006a, ApJS, 163, 1

Hopkins, P. F., Hernquist, L., Cox, T. J., \& Keres, D. 2007b, ApJ, submitted (astro-ph/0706.1243)

Hopkins, P. F., Hernquist, L., Cox, T. J., Robertson, B., \& Krause, E. 2007c, astro-ph/0701351

Hopkins, P. F., Hernquist, L., Cox, T. J., Robertson, B., \& Springel, V. 2006b, ApJS, 163, 50

Hopkins, P. F., Hernquist, L., Martini, P., Cox, T. J., Robertson, B., Di Matteo, T., \& Springel, V. 2005d, ApJ, 625, L71

Hopkins, P. F., Somerville, R. S., Hernquist, L., Cox, T. J., Robertson, B., \& Li, Y. 2006c, ApJ, 652, 864

Huang, S. \& Carlberg, R. G. 1997, ApJ, 480, 503

Hunter, D. A. \& Elmegreen, B. G. 2006, ApJS, 162, 49

Ibata, R., Chapman, S., Ferguson, A. M. N., Lewis, G., Irwin, M., \& Tanvir, N. 2005, ApJ, 634, 287

Ibata, R., Irwin, M., Lewis, G., Ferguson, A. M. N., \& Tanvir, N. 2001, Nature, 412, 49

Ibata, R., Martin, N. F., Irwin, M., Chapman, S., Ferguson, A. M. N., Lewis, G. F., \& McConnachie, A. W. 2007, astro-ph/0704.1318

Ibata, R. A., Irwin, M. J., Lewis, G. F., Ferguson, A. M. N., \& Tanvir, N. 2003, MNRAS, 340, L21

Jenkins, A., Frenk, C. S., White, S. D. M., Colberg, J. M., Cole, S., Evrard, A. E., Couchman, H. M. P., \& Yoshida, N. 2001, MNRAS, 321, 372

Kazantzidis, S. et al. 2007, in preparation

Kennicutt, Jr., R. C. 1989, ApJ, 344, 685

-. 1998, ApJ, 498, 541

Khochfar, S. \& Burkert, A. 2006, A\&A, 445, 403

Kregel, M., van der Kruit, P. C., \& de Grijs, R. 2002, MNRAS, 334,646

Lacey, C. \& Cole, S. 1993, MNRAS, 262, 627

McConnachie, A. W., Irwin, M. J., Ibata, R. A., Ferguson, A. M. N., Lewis, G. F., \& Tanvir, N. 2003, MNRAS, 343, 1335

McGaugh, S. S. \& de Blok, W. J. G. 1997, ApJ, 481, 689

Mihos, J. C. \& Hernquist, L. 1994, ApJ, 431, L9

Naab, T. \& Burkert, A. 2003, ApJ, 597, 893

Naab, T. \& Ostriker, J. P. 2006, MNRAS, 366, 899

Negroponte, J. \& White, S. D. M. 1983, MNRAS, 205, 1009

Newberg, H. J. et al. 2002, ApJ, 569, 245

Patterson, F. S. 1940, Harvard College Observatory Bulletin, 914, 9

Pérez, I. 2004, A\&A, 427, L17

Pohlen, M., Dettmar, R.-J., \& Lütticke, R. 2000, A\&A, 357, L1

Pohlen, M., Dettmar, R.-J., Lütticke, R., \& Aronica, G. 2002,

A\&A, 392, 807

Pohlen, M. \& Trujillo, I. 2006, A\&A, 454, 759

Pohlen, M., Zaroubi, S., Peletier, R. F., \& Dettmar, R. . 2007, MNRAS, 378, 594

Quinn, P. J. \& Goodman, J. 1986, ApJ, 309, 472

Quinn, P. J., Hernquist, L., \& Fullagar, D. P. 1993, ApJ, 403, 74

Roberts, M. S. \& Haynes, M. P. 1994, ARA\&A, 32, 115

Robertson, B., Cox, T. J., Hernquist, L., Franx, M., Hopkins, P. F., Martini, P., \& Springel, V. 2006a, ApJ, 641, 21

Robertson, B., Hernquist, L., Cox, T. J., Di Matteo, T., Hopkins,

P. F., Martini, P., \& Springel, V. 2006b, ApJ, 641, 90

Schaye, J. 2004, ApJ, 609, 667

Schmidt, M. 1959, ApJ, 129, 243

Schombert, J. M., McGaugh, S. S., \& Eder, J. A. 2001, AJ, 121, 2420 
Sellwood, J. A., Nelson, R. W., \& Tremaine, S. 1998, ApJ, 506, 590

Silk, J. \& Wyse, R. F. G. 1993, Phys. Rep., 231, 293

Somerville, R. S. \& Kolatt, T. S. 1999, MNRAS, 305, 1

Somerville, R. S., Lemson, G., Kolatt, T. S., \& Dekel, A. 2000, MNRAS, 316, 479

Spergel, D. N. et al. 2003, ApJS, 148, 175

Springel, V. 2005, MNRAS, 364, 1105

Springel, V., Di Matteo, T., \& Hernquist, L. 2005a, ApJ, 620, L79

-. 2005b, MNRAS, 361, 776

Springel, V. \& Hernquist, L. 2002, MNRAS, 333, 649

-. 2003, MNRAS, 339, 289

Thilker, D. A. et al. 2005, ApJ, 619, L79
Toomre, A. 1977, in Evolution of Galaxies and Stellar Populations, ed. B. M. Tinsley \& R. B. Larson, 401

Toomre, A. \& Toomre, J. 1972, ApJ, 178, 623

Trujillo, I. \& Pohlen, M. 2005, ApJ, 630, L17

van der Kruit, P. C. 1979, A\&AS, 38, 15

van der Kruit, P. C. 2001, in ASP Conf. Ser. 230: Galaxy Disks and Disk Galaxies, ed. J. G. Funes \& E. M. Corsini, 119-126

Velazquez, H. \& White, S. D. M. 1999, MNRAS, 304, 254

Walker, I. R., Mihos, J. C., \& Hernquist, L. 1996, ApJ, 460, 121

Wyse, R. F. G. et al. 2006, ApJ, 639, L13 